\title{
MedienPädagogik
}

Zeitschrift für Theorie und Praxis der Medienbildung

Jahrbuch Medienpädagogik 16:

Medienpädagogik in Zeiten einer tiefgreifenden Mediatisierung

Herausgegeben von Karsten D. Wolf, Klaus Rummler,

Patrick Bettinger und Sandra Aßmann

\section{Digital unterstütztes Lernen in der Pflegeausbildung}

\section{Die Care Reflection Online (CARO) - Lernumgebung}

\author{
Ingrid Darmann-Finck, Claudia Schepers, Karsten D. Wolf \\ und Jan Küster
}

\section{Zusammenfassung}

Die tiefgreifende Mediatisierung (Hepp 2018) schliesst nahezu alle gesellschaftlichen Bereiche ein, so auch das Gesundheitswesen. In der Pflegeausbildung ist das Potential des Einsatzes digitaler Medien noch nicht vollumfänglich ausgeschöpft. Zwar werden punktuell digitale Medien, wie etwa Lernplattformen (Moodle), Programme (PowerPoint) oder einzelne Methoden (flinga) in die Lehre integriert. Dennoch erfolgt der Einsatz selten pflegedidaktisch fundiert (Darmann-Finck 2010). Mediendidaktische Ansätze, anhand derer das digital unterstütze Lernen gestaltet werden kann (Kerres 2018; Jahnke 2015), adressieren die Anforderungen des Unterrichtsgegenstandes «Pflege» nicht ausreichend. Im Projekt CARO (Laufzeit 2016 bis 2019) wurde daher ein sog. Classroom-Learning-Interaction-System (CARO CLIS) zur Unterstützung von Live-Interaktionen im Klassenraum entwickelt und evaluiert. Das CARO CLIS unterstützt komplexe Lern-, Interaktions- und Reflexionsprozesse pflege- und mediendidaktisch fundiert. Im folgenden Beitrag wird sowohl die Entwicklung und Evaluation der Software als auch der Unterrichtsinhalte auf Basis des Design-based Research Ansatzes (Koppel 2016) aufgezeigt. Darüber hinaus wird exemplarisch dargestellt, wie mit dem CARO CLIS pflegedidaktisch fundierte komplexe Bildungsziele mit Hilfe mediendidaktischer Interaktionsformate im Unterricht umgesetzt werden können. 


\title{
Digitally supported learning in nursing training. The Care Reflection Online (CARO) learning environment
}

\begin{abstract}
The deep mediatization (Hepp 2018) integrates almost all sectors of society, including health care. In nursing training, the potential of the use of digital media has not yet been fully exploited. Digital media, such as learning platforms (Moodle), programs (PowerPoint) or individual tools (flinga), are integrated in teaching processes. Nevertheless, the use is rarely carried out in a nursingdidactically way (Darmann-Finck 2010). Media didactic approaches to shape digitally supported learning (Kerres 2018; Jahnke 2015), do not adequately address the requirements of contents in nursing training. In the CARO project (running 2016 to 2019), a so-called Classroom Learning Interaction System (CARO CLIS) was developed and evaluated to support live interactions in the classroom. The CARO CLIS supports complex learning, interaction and reflection processes in a nursing and media-didactic way. The following article shows the development and evaluation of the software as well as the teaching content based on the design-based research approach (DBR) (Koppel 2016). In addition, it is exemplified how the CARO CLIS supports nursing-didactically well-founded complex educational goals using media-didactic interaction formats.
\end{abstract}

\section{Ausgangslage}

In der Pflegeausbildung ist bisher kaum eine systematische Integration digital unterstützten Lernens zu verzeichnen (Kamin 2013). Lernprogramme, Lernplattformen (z. B. Moodle) oder Wissensdatenbanken (z. B. «Pflege kompakt») werden lediglich punktuell eingesetzt. Aus pflegedidaktischer Sicht beziehen sich die vorhandenen Lehr-/Lernangebote ausserdem nur auf ein äusserst begrenztes Spektrum an Bildungszielen (DarmannFinck 2010). Die Potenziale digital unterstützten Lernens sind bei weitem noch nicht ausgeschöpft. In der Mediendidaktik gibt es zwar eine Reihe didaktischer Ansätze, um digitales Lernen zu gestalten (vgl. z. B. Kerres 2018; siehe auch Jahnke 2015), diese reflektieren aber nicht die spezifischen Anforderungen des Unterrichtsgegenstands Pflege. Im BMBF- und 
ESF-geförderten Projekt CAre Reflection Online (CARO) werden daher pflege- und mediendidaktische Ansätze miteinander verknüpft und für die Pflegeausbildung fruchtbar gemacht. Ziel des Projekts ist es, eine pflege- und mediendidaktisch fundierte, digitale Lehr-/Lernumgebung zur Unterstützung von Lern-, Interaktions- und Reflexionsprozessen für die Pflegeausbildung zu entwickeln. Um die inhaltlichen Lernangebote und die digitale Lehr-/Lernumgebung auf die Anforderungen der Pflegeschulen und der potentiellen Nutzerinnen und Nutzer abstimmen zu können, erfolgt im Projekt eine fortlaufende kleinschrittige Evaluation im Stile des Design Based Research Ansatzes (Koppel 2016).

Die CARO-Lehr-/Lernumgebung kann anhand der Elemente von (digitalen) Lehr-/Lernangeboten «Content», «Communication» und «Construction» (Kerres 2018, 432 ff.) beschrieben werden: Im Rahmen des Projekts werden Lehr-/Lernangebote zu drei thematischen Bereichen entwickelt, nämlich zur Pflege von Menschen mit Demenz im Krankenhaus, zum freiheitsförderlichen pflegerischen Handeln und zur Transkulturellen Pflege. Die Lehr-/Lernangebote beziehen sich dabei jeweils auf unterschiedliche didaktische Ziel- bzw. Bildungsdimensionen. In Abgrenzung zu rein expositorischen e-learning-Konzepten, die nur wenig Interaktion inkludieren, bietet die CARO-Anwendung neben der Bereitstellung von Inhalten (content) einen funktionalen Fokus auf das aktive Management von Kommunikation (communication) zwischen Lehrpersonen sowie Schülerinnen und Schülern im Pflegepräsenzunterricht sowie die digitale Unterstützung des selbständigen problem- bzw. fallbasierten generativen Lernens in Gruppen (construction).

Konkret können die Lehrpersonen über ein Dashboard zunächst den Unterrichtsverlauf planen, auf vorgefertigte Unterrichtsmaterialien und -pläne zugreifen sowie diese individuell mit Material sowie Aufgaben ergänzen. Von der Unterrichtsplanung ausgehend haben die Lehrpersonen bei der Unterrichtsdurchführung im Klassenraum die Möglichkeit, phasenweise Arbeitsaufträge für die «CARO Schüler*innen App» freizuschalten, die von den Schülerinnen und Schüler erstellten Arbeitsergebnisse anzuschauen, diese zu bewerten und zu verwalten oder sie im Plenum mittels eines Präsentationsboards per Beamer oder Interactive Whiteboard zur Diskussion zu stellen. 
Die kompetenzfördernde Aktivierung der Schülerinnen und Schüler erfolgt über die mobile «CARO Schüler*innen App»: die Arbeitsaufträge können didaktisch variantenreich gestaltet werden, damit die Schülerinnen und Schüler sich - einzeln oder in Gruppen - aktiv die Inhalte erarbeiten und auch kooperativ mit pflegedidaktischen Fällen und Problemstellungen arbeiten können (construction). Die mobile CARO-App kann somit auch in einem «Bring Your Own Device-Konzept» genutzt werden, bei dem die Schülerinnen und Schüler ihre eigenen mobilen Endgeräte wie z. B. Smartphones oder Tablets nutzen. Natürlich können auch von der Schule gestellten Geräte eingesetzt werden.

In diesem Beitrag werden Methoden und ausgewählte Ergebnisse der formativen Evaluation der CARO-Lehr-/Lernumgebung vorgestellt. Die Evaluation richtet sich auf die Optimierung der drei Bestandteile von (digitalen) Lehr-/Lernangeboten, nämlich der Inhalte (content), der unterrichtlichen Interaktion (communication) und der generativen Lernaktivitäten durch die Schülerinnen und Schüler (construction). Sie bezieht sich in allen drei Punkten sowohl auf die technische (Entwicklung und Programmierung) als auf die inhaltliche Ebene (Entwicklung von Lehr-Lernmaterial) sowie auf das Zusammenspiel beider Ebenen. Es sollen Anpassungen ermittelt werden, die erforderlich sind, um das Fördern der pflegedidaktischen Bildungsziele bestmöglich unterstützen zu können. Im Mittelpunkt des Beitrags steht der erste Feldtest.

Vor der Darstellung der Methoden und ausgewählter Ergebnisse erfolgt eine kurze Erläuterung der pflegedidaktischen Grundlagen sowie der mediendidaktischen Gestaltung der CARO-Lehr-/Lernumgebung. Der Beitrag endet mit einem Ausblick.

\section{Pflegedidaktische Grundlagen}

Die CARO-Lehr-/Lernumgebung ist mit dem Ziel entwickelt worden, die Interaktionistische Pflegedidaktik, einen bildungstheoretisch fundierten didaktischen Ansatz, der sich in Anlehnung an Klafki an «Bildung als Zielkategorie von beruflichen Lehr-/Lernprozessen» orientiert (Darmann-Finck 2010, 157), zu unterstützen. Drei zentrale Ziel- bzw. Bildungsdimensionen stehen im Mittelpunkt des Modells. Neben (1) dem problemlösenden Denken auf der Basis von (evidenzbasiertem) Regelwissen, sollen (2) die 
Fähigkeiten zum Selbst- und Fremdverstehen sowie zur kommunikativen Verständigung und (3) zur kritischen Reflexion durch Denken in Widersprüchen gefördert werden. In der Pflegeausbildung sollen die Schülerinnen und Schüler befähigt werden, zu diesen Ziel- bzw. Erkenntnisdimensionen die jeweils passenden Erkenntnismethoden anzuwenden. Für die Entwicklung von Lehr-/Lernangeboten bedeutet dies, dass der Unterrichtsgegenstand im Hinblick auf mögliche Erkenntnisdimensionen ausgelegt werden muss bzw. solche Unterrichtsgegenstände ausgewählt werden, die die Aneignung der Bildungsdimensionen ermöglichen. Ausserdem sollen solche Unterrichtsmethoden eingesetzt werden, die geeignet sind, die für eine Bildungsdimension spezifischen Erkenntnisse zu generieren. Erkenntnisinteresse und Erkenntnismethoden müssen im Unterricht gut aufeinander abgestimmt werden. Im Sinne eines kompetenzorientierten Unterrichts werden im Rahmen des Projekts Lernsituationen entwickelt, die jeweils mit einer komplexen, filmisch dargestellten Fallsituation starten, die auf einem Schlüsselproblem der Berufswirklichkeit (Klafki 1994; Darmann-Finck 2010, 186) basiert. Zur Identifikation geeigneter Bildungsziele und -Inhalte kann die pflegedidaktische Heuristik (Darmann-Finck 2010, 169ff.) direkt während der Konzeption von Unterricht in CARO genutzt werden, da diese in der CARO-Lehr-/Lernumgebung in einer Curriculum-Planungs-Anwendung (s.u.) hinterlegt ist.

In der Interaktionistischen Pflegedidaktik wird Unterricht des Weiteren als ein Prozess der Verständigung über Bedeutungen analysiert und konzipiert (Darmann-Finck 2010, 158 ff.). Lehrpersonen und Schülerinnen und Schüler tragen unterschiedliche Bedeutungen an den Unterrichtsgegenstand heran. Vor dem Hintergrund eines interaktionistischen bzw. konstruktivistischen Lehr-/Lernverständnisses besteht die Aufgabe der Lehrpersonen darin, die Schülerinnen und Schüler durch das Lehr-/Lernarrangement anzuregen, sich erkenntnisfördernde Fragen zu stellen, die eigenen Bedeutungen zu überprüfen und ggf. zu modifizieren oder aber auch bewusst beizubehalten. Schülerinnen und Schüler sind demzufolge ebenso wie Lehrpersonen als Subjekte im Lehr-Lernprozess zu betrachten, die über umfangreiches Wissen verfügen und im unterrichtlichen Aushandlungsprozess über Bedeutungen sowohl Gestalterinnen und Gestalter als auch Rezipientinnen und Rezipienten sind (Darmann-Finck 2010, 161; Meyer 2008, 121). 


\section{Mediendidaktische Gestaltung der CARO Lehr-I Lernumgebung}

Für die Planung des Gesamtsystems wurden zunächst auf Basis der drei Digital Didactical Design Layers (Jahnke 2015, 133; siehe Abb. 1) die grundlegenden Anforderungen an das Gesamtsystem analysiert und festgeschrieben:

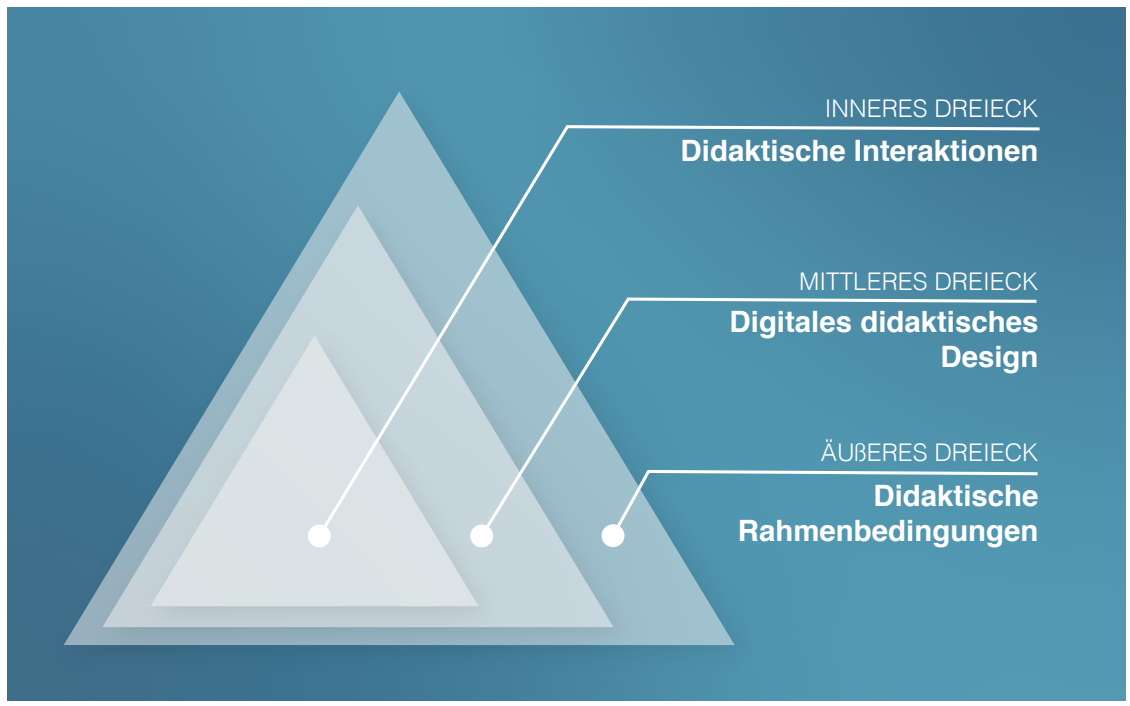

Abb. 1.: Digital Didactics Layers nach Jahnke (2015) (eigene Darstellung).

(1) Die didaktischen Rahmenbedingungen (didactical conditions) werden durch die Anforderung sowohl der Schul- als auch der Berufspraxis im Rahmen der dualen Pflegeausbildung inkl. der Lehrpersonenaus- und -fortbildung sowie der vorhandenen bzw. fehlenden digitalen Kompetenzen und Ressourcen der Lernenden, der Lehrenden und der Schulen definiert. Für die Gestaltung von CARO war dabei insbesondere wichtig, dass das zu konzipierende System möglichst flexibel durch die Lehrenden selbst an jeweils spezifische Rahmenbedingungen anzupassen ist. Konkret sollte das System ermöglichen, auch kleinere Einheiten aus grösseren Unterrichtseinheiten einsetzen zu können und diese beliebig zu erweitern, verändern oder auch verkürzen zu können. Dies soll auch digitalen Laien ohne besondere Vorkenntnisse möglich sein. Weiterhin ist es notwendig, 
trotz einer Vorplanung flexibel in Unterrichtssituationen zu reagieren und Abläufe im Prozess ändern zu können. Da die digitale Ausstattung in der Pflegeausbildung sehr heterogen ist, sollte das System auch mit (privaten) mobilen Endgeräten der Beteiligten (Bring Your Own Device-Konzept) einsetzbar sein, was ein responsives Design der Anwendung für unterschiedliche Geräte (Smartphones, Tablets, Labtops) erfordert.

(2) Das digitale didaktische Design (digital didactical design) basiert auf den im Projekt entworfenen Curricula (Kompetenzen, Inhalte) zu drei ausgewählten Themen der Pflegeausbildung ${ }^{1}$ sowie dem mediendidaktischen Planungsansatz nach (Kerres 2018). Die Kompetenzen und Lerninhalte adressieren demnach unterschiedliche Bildungsdimensionen (s. Kapitel 2) und Kompetenzniveaus. Der Schwerpunkt der verwendeten didaktischen Methoden liegt durch das Verständnis von Unterrichtsmethoden als Erkenntnismethoden eher auf exploratorischen und problem-/ bzw. fallbasierten Schemata in kooperativen Lernsettings, ohne auf expositorische Vermittlungsphasen völlig zu verzichten. Das Design wird im Klassenunterricht (Face-to-Face Interaktion) durchgeführt, optionale Elemente des Blended Learnings (Mischung von Online-Interaktion und Face-to-Face Interaktion) sind über Arbeitsaufträge (Haus-, bzw. Praxisaufgaben) denkbar. Diese didaktischen Anforderungen erfordern somit neben expositorischen Selbstlerneinheiten insbesondere eine Unterstützung kommunikativer, kooperativer sowie kollaborativer Lehr-Lern-Prozesse im Sinne einer konstruktionistischen Lehr-Lern-Umgebung (Wolf 2003, 143 ff.). Die Komplexität der curricularen Ziele erfordert dabei phasenweise die Unterstützung und Moderation durch Lehrpersonen. Ein zentrales Konzept von CARO ist deshalb die digitale Unterstützung von Präsenzunterricht bzw. synchronen Fernunterrichts per Videokonferenz.

(3) Die digitalen Interaktionen (digital interactions) werden auf der Basis von Kurs- und Seminarmethoden zur Förderung des aktiven Lernens konzipiert (Knoll und Knoll 2007; Weidenmann 2015; Prince 2004). Dabei liegt der Hauptfokus der digitalen Unterstützung darin, die Durchführung der

1 Siehe dazu auch das Nationale Mustercurriculum Kommikative Kompetenz in der Pflege NaKOMM (http://nakomm.ipp.uni-bremen.de). 
jeweiligen Methode organisatorisch und medial so zu unterstützen, dass die Lehrenden sich möglichst vollständig auf die Moderation und Unterstützung konzentrieren können. Auf diese Weise soll die nutzbare Lernzeit in hohem Masse ausgeschöpft und möglichst alle Lernenden in der Lerngruppe aktiviert werden. Die Abbildung 2 stellt die digitale Unterstützung einer typischen Unterrichtssequenz «Kritische Reflexion von Widersprüchen» mit Hilfe der CARO Umgebung schematisch dar.

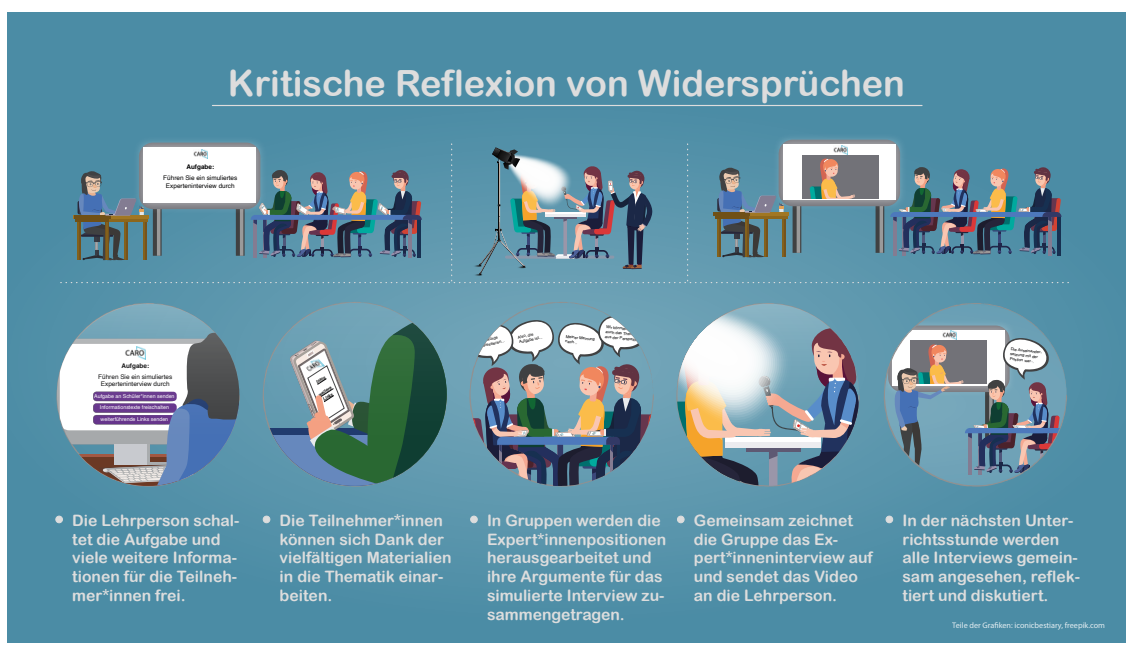

Abb. 2.: Schematische Darstellung einer mit CARO unterstützten Unterrichtssequenz (eigene Darstellung).

Um diesen Anforderungen zu entsprechen, erstreckt sich die Architektur der CARO Umgebung über einen Verbund von Anwendungen:

(1) Curriculum Authoring Application (CAA) «CARO Curriculum»: In der CAA erstellt das CARO Autorinnen und Autorenteam auf der Basis des Pflegecurriculums Musterinhalte für den Unterricht. Dabei stehen dem Autorinnen und Autorenteam entsprechende Editorenmodule zum Einpflegen der Heuristik (siehe Abschnitt 2 Pflegedidaktische Grundlagen), Kompetenzen, Lernsituationen, Lernsequenzen und Unterrichtsmaterialien zur Verfügung. 
(2) Teacher Classroom Application (TCA) «CARO Lehrer*innen App»: In der TCA bereiten die Lehrpersonen auf Basis der Musterinhalte ihren Unterricht vor (Vorbereitungsphase). Dabei können sie den Verlauf anpassen und Material über die Editorenmodule verändern oder ergänzen. In der Durchführungsphase steuern sie zudem live im Unterricht den Unterrichtsverlauf und die Interaktionen (Arbeitsaufträge) mit und zwischen den Schülerinnen und Schülern. In der TCA können die Lehrpersonen des Weiteren die Bearbeitungsschritte der Schülerinnen und Schüler kontrollieren. Arbeitsergebnisse können für das Plenum freigegeben werden.

(3) Public Display Application (PDA) «CARO Beamer App»: Die PDA wird idealerweise per Beamer oder auf einem Interactive Whiteboard im Klassenraum dargestellt. In der PDA können die Lehrpersonen für die TCA beliebig die Ergebnisse der Einzel- oder Gruppenarbeiten sowie andere Lehrmaterialien darstellen. Dabei sind weitere Live-Interaktion möglich, wie z. B. das Clustern von Abfragekarten durch Drag-and-Drop. So können die Ergebnisse im Klassenverbund gemeinsam und interaktiv reflektiert werden, die aufbereiteten und ggf. gemeinsam veränderten Ergebnissicherungen können als digitale Artefakte persistent zur Dokumentation gespeichert werden.

(4) Learner Interaction Application (LIA) «CARO Schüler*innen App»: Die Schülerinnen und Schüler nutzen die LIA auf mobilen Endgeräten, um auf die Lernmaterialien und -interaktionen zuzugreifen. Dazu wird die TCA der Lehrpersonen über ein Publication-Subscription Modell (Birman und Joseph 1987; Meteor Development Group 2019) mit der LIA auf den Geräten der Schülerinnen und Schüler synchronisiert. Die Lehrpersonen können somit steuern, welche Informationen bzw. Interaktionen gerade für die Schülerinnen und Schüler aktiv zugänglich sind.

Die hier beschriebene Kombination aus Content Authoring und Classroom Management in der Teacher Classroom Application (TCA), Classroom Interaction in der Learner Interaction Application (LIA) sowie öffentlicher Bereitstellung von Arbeitsergebnissen und Moderationsunterstützung in der Public Display Application (PDA) ermöglicht eine digital unterstützte 
Live-Interaktion im Klassenraum, weshalb wir das Gesamtsystem als ein Classroom Learning Interaction System (CLIS) bezeichnen (Cao, EspondaArgüero, und Rojas 2016). Die Softwarearchitektur ist als ein Anwendungsverbund auf Basis von Node.js (nodejs.org) und dem Full-stack JavaScript Framework Meteor (meteor.com) realisiert (siehe Abb. 3).

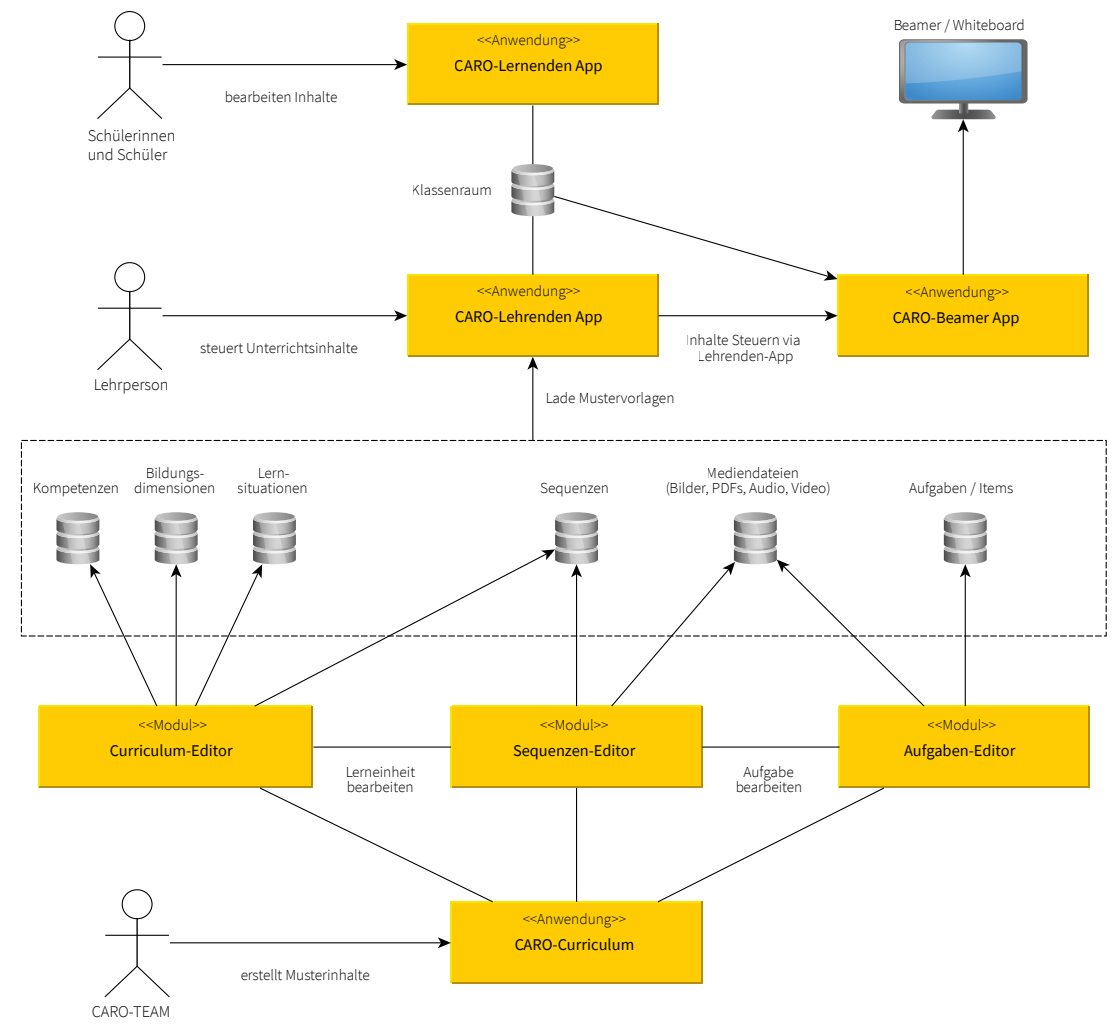

Abb. 3.: Übersicht der CARO-Gesamtarchitektur.

\section{Entwicklung und Evaluation der Softwareumgebung sowie der Unterrichtsinhalte}

Um eine agile Entwicklung der Softwareumgebung unter Einbezug einer qualitativ hochwertigen und der Anwendung angemessenen Evaluation zu realisieren, wurde methodologisch der Design Based Research - Ansatz 
(DBR) zugrunde gelegt. Dieser umfasst eine systematische Dokumentation der Evaluationsergebnisse, die Einbeziehung potentieller Nutzerinnen und Nutzer von Beginn an sowie eine iterative Vorgehensweise (vgl. Koppel 2016). Das methodische Vorgehen im Sinne des DBR gliedert sich in vier Phasen: I) Problemanalyse, II) Konzeption, III) Konzeptevaluation sowie IV) Reflexion (vgl. Akker u. a. 2006, 96; siehe Abbildung 4).

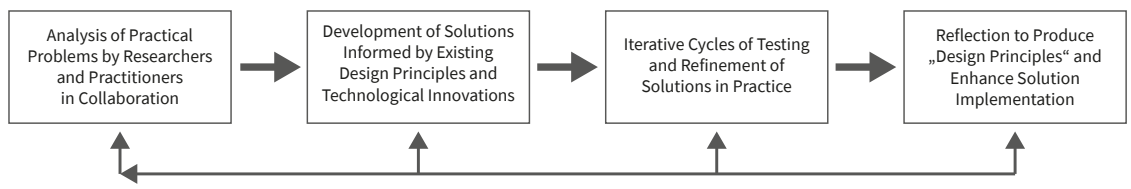

Abb. 4.: Vier Phasen des DBR-Prozesses nach Reeves (Koppel 2016, 151).

In jeder dieser Phasen werden Designzyklen durchlaufen, die anhand der Ebenen Gestaltung, Durchführung, Überprüfung und Re-Design konzipiert wurden (siehe Abb. 4). Anhand dieser Ebenen konnten Evaluationszyklen stringent strukturiert und Evaluations-Teilergebnisse zielführend aufeinander bezogen werden. Indem die Anwendung bereits während des Entwicklungsprozesses von den potentiellen Anwenderinnen und Anwender getestet wurde, konnten die Anforderungen der Unterrichtspraxis frühzeitig erfasst und bei der Entwicklung berücksichtigt werden.

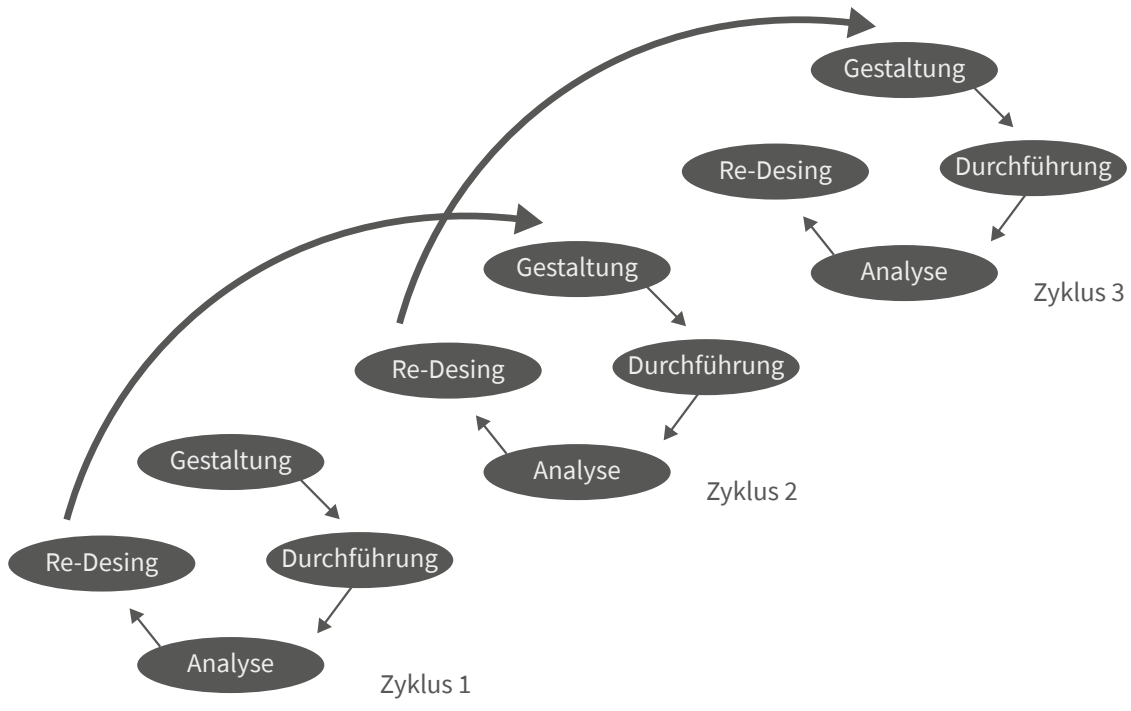

Abb. 5.: Schematischer Ablauf DBR-Zyklen (Koppel 2016, 154). 
Im Projekt CARO wurden bereits in der Phase der Problemanalyse (I) unterschiedliche Designzyklen durchgeführt, um das Konzept der CAROAnwendung auszudifferenzieren. In den ersten zwei Zyklen (Zeitraum: Februar bis Mai 2017) wurden mit Hilfe eines Paper Prototype-Tests und eines Cognitive Walkthrough einfache Systemanwendungen getestet. Der Evaluationsgegenstand bestand lediglich in einem papierbasierten Konzept. In der II. Phase, der Konzeption, wurde das Forschungsdesign für das gesamte Projekt konkretisiert (Planung der ersten Feldtests, Abstimmung der Erhebungsmethoden, Absprache mit den Praxispartnerinnen und Partner), ausserdem erfolgte eine Feinabstimmung der technischen mit der inhaltlichen Gestaltung. Die Anwendung lag in einem sehr einfachen Entwicklungsstadium vor und konnte im geschützten Rahmen eines Workshops mit Lehrpersonen der beteiligten Modellschulen erprobt werden. Sie wurde daraufhin weiterentwickelt und befand sich am Ende der II. Phase in einem so weit fortgeschrittenen Entwicklungsstadium, dass sie in Feldtests getestet werden konnte.

In der III. und IV. Phase, der Konzeptevaluation und Reflexion wurden in kleinschrittigen Designzyklen erste Feldtests durchgeführt, evaluiert und Schlussfolgerungen hinsichtlich erforderlicher inhaltlicher und technischer Anpassungen gezogen. In den Feldtests wurden Unterrichte mit der CARO Anwendung durchgeführt. Im Rahmen der Konzeptevaluation wurden bis Ende 2019 zehn Feldtests durchgeführt. Für den ersten Feldtest musste das bis dato entwickelte technische System mit den vorgesehenen Inhalten zusammengeführt werden. In diesem Zyklus erfolgten damit erstmals eine Integration und Abstimmung der technischen und inhaltlichen Ebene.

Um das Vorgehen zu veranschaulichen, wird im Folgenden anhand der Designzyklen Gestaltung, Durchführung, Analyse, Re-Design (siehe auch Abb. 5) exemplarisch der erste Feldtest dargestellt.

Gestaltung: Das System erfüllte zum Zeitpunkt der ersten Testung folgende Kriterien: Auf der inhaltlichen Ebene waren die Fachinhalte einer Lerneinheit (Theorie der besonderen Ungewissheit im pflegerischen Handeln, Evers 2012) inkl. Arbeitsaufträgen und Materialien (Dokumente, Links) in die Datenbank des Systems eingepflegt und somit Bestandteil 
des CARO Unterrichts. In technischer Hinsicht konnten die Lehrpersonen Schülerinnen und Schüler über eine Transaktionsnummer (TAN) in einen privaten digitalen Klassenraum innerhalb der CARO App einladen. Die Anwendung war von einem Browser aus auszuführen, eine Installation war nicht erforderlich. Zu diesem Zeitpunkt konnte eine vollständige Lerneinheit (4 Unterrichtsstunden, 180 Minuten) durchgeführt werden. Verschiedene mediale Formate, wie Videos (eine filmisch dargestellte Fallsituation der Berufswirklichkeit und ein Experteninterview), Arbeitsblätter (z. B. Erstellung eines Mindmaps), waren in die Lehr-/Lernumgebung eingebunden, ausserdem konnten verschiedene Antwortformate über die «CARO Schüler*innen App» bedient werden, wie Freitextantworten, Skaleneingabe (via Range-Slider) oder Tabelleneintragungen.

Durchführung: Der erste Feldtest fand in einem Seminar des Studiengangs BA Pflegewissenschaft-dual, Schwerpunkt Lehre an der Universität Bremen statt. Teilgenommen haben 16 Studierende. Bei der technischen Umsetzung wurde die Lehrende von einem Kollegen aus der Mediendidaktik unterstützt. Vier wissenschaftliche Mitarbeiterinnen und Mitarbeiter sowie eine Praktikantin protokollierten den Testdurchlauf. Der Unterricht dauerte etwa fünf Zeitstunden (von 8:18 Uhr bis 13:00 Uhr).

Datenerhebung und -auswertung: Für die Datenerhebung kamen im Rahmen der ersten Testung nicht-teilnehmende Beobachtungen mit Dokumentation mittels teilstandardisierter Beobachtungsprotokolle (vgl. Flick, Kardorff, und Steinke 2017, 396) zur Anwendung. Zudem wurde der Feldtest auf Video aufgezeichnet. Des Weiteren erfolgte am Schluss des Unterrichts eine mündliche Abfrage bei den Teilnehmenden hinsichtlich der Zufriedenheit mit dem Lernangebot. In weiteren Testungen wurden im Sinne eines Mixed-Methods-Design (Kelle und Erzberger 2007) weitere Daten erhoben, nämlich qualitative Interviews mit Lehrpersonenn sowie standardisierte Befragungen der Studierenden in ihrer Rolle als Schülerinnen und Schüler. Die Prozesse der Datenerhebung und -auswertung waren durch das iterative Vorgehen eng miteinander verschränkt. Die Beobachtungsprotokolle wurden in Anlehnung an die qualitative Inhaltsanalyse ausgewertet (Kuckartz 2016; Mayring 2015). In der weiteren Analyse der 
Protokolle erwiesen sich die drei Kategorien didaktischer Komponenten von Lehr-Lern-Arrangements (Kerres und Witt 2003) «Content», «Communication» und «Construction» als passend für die Sortierung der Codes. Die aus den Beobachtungsprotokollen gewonnenen Kategorien wurden im Projektteam diskutiert und auf Relevanz für den weiteren Entwicklungsprozess geprüft, so dass notwendige technische und inhaltliche Anpassungen vorgenommen werden konnten. Die drei Kategorien «Content», «Communication» und «Construction» leiteten auch den weiteren Evaluationsprozess. Die im Rahmen der ersten Testung gewonnenen zentralen Befunde innerhalb dieser Kategorien werden im Folgenden ebenso beschrieben wie auch die Schlussfolgerungen für das Re-Design, die daraus gezogen wurden.

\section{Ergebnisse}

Der Schwerpunkt der Ergebnisdarstellung liegt zwar auf den Ergebnissen der Evaluation des ersten Feldtests, wird aber zum Teil noch erweitert um die Ergebnisse weiterer Tests. Die Darstellung gliedert sich anhand der drei oben genannten Kategorien didaktischer Komponenten von LehrLern-Arrangements (Kerres und Witt 2003).

\subsection{Content: Reduktion und Flexibilisierung}

Hinsichtlich des Contents ergab die Evaluation, dass der Umfang an dargebotenen Inhalten zu gross war. Folgende von der CARO-Lernumgebung vorgehaltenen Inhalte wurden als zu umfassend identifiziert:

- Lehr-Lernmaterial, das den Schülerinnen und Schüler in Form von Inputs direkt in der "CARO Schüler*innen App» angeboten wurde, wie z. B. Texte und Videos;

- Vorbereitungsmaterial für Lehrpersonen, wie z. B. weiterführende Literatur (bspw. zum Thema «Pflege von Menschen mit Demenz als Nebendiagnose im Krankenhaus»);

- ausführliche Artikulationsschemata für die Lehrpersonen. 
Aus dieser Feststellung wurde der Schluss gezogen, die Inhalte deutlich zu reduzieren. Nach Möglichkeit sollte der Gültigkeitsumfang der Grundaussagen dabei nicht eingeschränkt werden (Arnold 1990, 60).

Als weiteres wesentliches Ergebnis der Evaluation stellte sich heraus, dass die ursprüngliche Planung, komplette Lernsituationen im Umfang von ca. 25 UStd. zu entwickeln, die mit einem vorgegebenen Ablauf kompakt unterrichtet werden sollten, unpraktikabel war und nicht den Anforderungen der Pflegeschulen entsprach. Im Rahmen der ersten und der weiteren Testungen erwies sich, dass es eher möglich ist, kleinere Einheiten in die schon vorhandenen Curricula zu integrieren und an die jeweiligen Lernvoraussetzungen der Schülerinnen und Schüler anzupassen. Entsprechend dieser Einsicht wurde das inhaltliche Material erneut gesichtet und eine neue Struktur des Lehr-/Lernangebots konzipiert. Ausgehend von einer filmisch dargestellten Fallsituation wurden kleinere Unterrichtseinheiten für eine oder zwei in sich abgegrenzte Doppelstunde/n entwickelt, die auch unabhängig voneinander unterrichtet werden können. Lehrpersonen haben so die Möglichkeit, nach dem «Baukastenprinzip» einzelne CARO-Unterrichtsstunden in ihren Gesamtunterricht einzupassen. Sie können beispielsweise gezielt Unterrichtseinheiten zu bestimmten Themen oder zu verschiedenen Bildungs- und Erkenntnisdimensionen, einzelne Arbeitsaufträge, oder das zur Verfügung stehende Lehr-Lernmaterial für die individuelle Unterrichtsplanung nutzen.

Werden in der CARO-Lehr-/Lernumgebung derzeit noch überwiegend die zu lernenden Inhalte anhand von Lern- und Arbeitsmaterialien vorgegeben, so bietet CARO auch die Möglichkeit, dass die Schülerinnen und Schüler selbstständig Antworten auf komplexe pflegerische Problemstellungen z. B. im Internet recherchieren oder ihre persönlichen Netzwerke in den Lernprozess einbeziehen. Durch die Möglichkeit das Internet sowie Funktionen von CARO im Präsenzunterricht miteinander zu verknüpfen und für Lernprozesse nutzbar zu machen, eröffnen sich neue Lernräume, sog. «CrossActionSpaces» (Jahnke 2015, 2). 


\subsection{Communication: Verknüpfung digitaler und analoger Kommunikation}

Als weiteres zentrales Ergebnis im Rahmen der Evaluation wurde festgestellt, dass sich im Vergleich zum traditionellen Unterricht durch den digital unterstützen Unterricht die Unterrichtsinteraktion verändert. Kennzeichnend für die Unterrichtsinteraktion mit der CARO Lehr-/Lernanwendung ist die Verknüpfung von digitaler und analoger Kommunikation. Im Rahmen der Testungen konnte beobachtet werden, dass die Lehrpersonen sich zum Teil darauf verlassen haben, dass die digitale die analoge Interaktion ersetzt. Um die Potenziale der CARO-Lehr-/Lernumgebung aber tatsächlich optimal nutzen zu können, müssen sich die Lehrpersonen bewusst machen, an welchen Stellen sie den Lernprozess durch analoge Kommunikation unterstützen müssen.

Diese Schlussfolgerung soll anhand von zwei Beispielen verdeutlicht werden, nämlich an der Gestaltung des für Unterrichtsgespräche typischen Dreischritts «Initiation - Reply - Evaluation» (Mehan 1979, 52) und der expliziten Versprachlichung der Lehrpersonenaktivitäten in der «CARO Lehrer*innen App» sowie der Interaktionen in der «CARO Schüler*innen App».

\subsubsection{Explizite Versprachlichung}

Eine stark lehrpersonenzentrierte Steuerung des Unterrichtsgesprächs durch das Stellen von Lehrpersonenfragen und die falsifizierende oder verifizierende Bewertung der Schülerinnen- und Schülerantworten wird aus interaktionstheoretischer Sicht als wenig lernförderlich bewertet. Lehrpersonen sollen Unterrichtsgespräche vielmehr so gestalten, dass die dynamische Spannung zwischen der Bedeutung, die die Schülerinnen und Schüler dem Unterrichtsgegenstand zuschreiben, und der von den Lehrpersonen intendierten Bedeutung aufrechterhalten wird, um eine eigenständige Wissenskonstruktion der Schülerinnen und Schüler zu ermöglichen (Voigt 1990). Dies gelingt, indem sich Lehrpersonen mit richtigen Lösungen zurückhalten und die Schülerinnen und Schüler auffordern, selbst Bewertungen vorzunehmen, indem sie ungewöhnliche Gedanken von Schülerinnen und Schüler stärken oder indem sie bei unterschiedlichen 
Lösungsansätzen der Schülerinnen und Schüler Differenzen transparent machen und sie als Lernimpulse nutzen (Darmann-Finck 2010, 160). Im digital unterstützen CARO-Unterricht erfolgt der Impuls von Seiten der Lehrpersonen («Initiation») i.d.R. durch eine über die App übermittelte Aufgabenstellung, die von den Schülerinnen und Schülern zu bearbeiten ist. Die Arbeitsergebnisse («Reply») aller Schülerinnen und Schülern können im Anschluss z. B. auf dem Whiteboard sichtbar gemacht werden. Damit steht die Antwort der einzelnen Schülerinnen und Schülern für die Gesamtgruppe zur Bewertung zur Verfügung. Das Potenzial zur Erweiterung des Unterrichtsgesprächs soll beispielhaft erläutert werden.

Im Rahmen der Unterrichtseinheit «Herausforderung Kommunikation» (Lerninsel «Ungewissheit im pflegerischen Handeln») werden Kommunikationsformen erarbeitet, die zum Ziel haben, die soziale und personale Identität der zu pflegenden Personen mit Demenz zu fördern und das Person-Sein zu stärken (Deutsches Netzwerk für Qualitätsentwicklung in der Pflege (DNQP) 2019). Die Lehrperson schaltet nach einer kurzen Einführung in die Thematik einen Arbeitsauftrag frei. Der Arbeitsauftrag knüpft an das Vorwissen der Schülerinnen und Schüler an, sie werden aufgefordert, Regeln, die sie zur Kommunikation mit Menschen mit Demenz kennen, festzuhalten.

Die Schülerinnen und Schüler geben ihre Antworten in die App ein und senden sie zurück. Die Lehrperson sammelt die Antworten und kann diese entweder zunächst selbst auf dem eigenen Laptop bewerten und systematisieren und dann der Gesamtgruppe das aufbereitete Ergebnis zur Verfügung stellen, oder sie kann die gesammelten Arbeitsergebnisse der Schülerinnen und Schüler gemeinsam mit der Klasse auswerten, indem sie sie per Beamer oder Whiteboard präsentiert. Die Freitextantworten können geclustert werden, indem zu verschiedenen Kategorien jeweils Tabellenspalten generiert werden, in die die «Karten» mit den Schülerinnen und Schüler-Antworten einsortiert werden können. Die Lehrperson könnte das Clustern auch im Dialog mit den Schülerinnen und Schülern vornehmen, indem jede «Karte», also jede einzelne Schülerinnen und Schüler-Antwort zunächst einzeln auf das Board gezogen und dann diskutiert und zugeordnet wird. Es ist davon auszugehen, dass die von den Schülerinnen und Schülern artikulierten Regeln nicht dem aktuellen Stand des Wissens 
entsprechen. Anstelle, dass die Lehrpersonen die Antworten bewertet, könnten die Schülerinnen und Schüler, wenn alle Antworten sichtbar sind, aufgefordert werden, sich gegenseitig nach Begründungen für die genannten Kommunikationsregeln fragen. Dabei werden sich voraussichtlich Wissenslücken ergeben. Die Lehrperson gibt im Anschluss an diese Sequenz einen Ausblick auf die folgende Unterrichtseinheit, in der wissenschaftlich fundierte Ansatzpunkte zur Kommunikation mit Menschen mit Demenz erarbeitet werden. Sie kündigt an, dass die Vorschläge der Schülerinnen und Schülern nach Abschluss der Einheit vor dem Hintergrund der neu erworbenen Kompetenzen überprüft werden.

Indem im CARO-Unterricht die Antworten aller Mitschülerinnen und Mitschülern für das Plenum sichtbar gemacht werden können, ergibt sich das Potenzial, die Phase der Bewertung an die Schülerinnen und Schüler zurückzugeben und dadurch höhere Denk- und Lernprozesse zu fördern.

Sind wie bei diesem Beispiel in Bezug auf die Fragen nach Begründungen für Kommunikationsregeln längere Antworten notwendig, mit denen etwa komplexere Zusammenhänge, Reflexionen oder Argumentationen dargestellt werden sollen (Kerres 2018, 433), sind digitale Abfrageformate weniger geeignet, weil es nicht praktikabel ist, die Antworten aller Schülerinnen und Schülern auszuwerten. Unterrichtsgespräche mit diesen Gegenständen müssen daher entweder in analoge oder digitale Kleingruppen verlagert oder aber sie müssen analog im Plenum geführt werden. Im Rahmen des Re-Designs wurde daher bei der Unterrichtsentwicklung unterschieden, welche Lernziele durch digital unterstützte und welche durch analoge Lernangebote gefördert werden sollen. Der Kompetenzerwerb findet durch eine sinnvolle Kombination beider Informationsformen statt.

\subsection{Interaktionen in der "CARO Schüler*innen App»}

Neben der inhaltlichen Erarbeitung dient die unterrichtliche Interaktion ausserdem der Steuerung des Unterrichtsprozesses. Im CARO-Unterricht erhalten die Schülerinnen und Schüler per App Informationen hinsichtlich der nächsten Unterrichtsschritte. Bei der Evaluation hat sich herausgestellt, dass die Instruktionen zum Teil nicht genügend erläutert wurden und dass die Lernenden im Ungewissen waren, welche Schritte als 
nächstes geplant sind bzw. über welche Informationen die Lehrperson auf ihrem Rechner verfügt. Typische Fragen der Schülerinnen und Schülern lauteten während des ersten Feldtests etwa, «Können Sie auch sehen, was wir jetzt verfasst haben?» oder «Müssen wir auf 〈fertig〉 klicken, wenn wir fertig sind?» Aus diesem Befund wurde der Schluss gezogen, dass die Lehrperson insbesondere die Übergänge im unterrichtlichen Geschehen auch explizit verbalisieren sollte, um den Prozess auf diese Weise transparent zu steuern und die Schülerinnen und Schüler durch den digital unterstützten Unterricht zu leiten. Die Moderation der Lehrperson kann hierbei die «Selbstbeschreibungsfähigkeit» der Anwendung unterstützen. Ein Konzept zur Verbesserung der gesamten Usability wird aktuell erstellt und schrittweise umgesetzt.

Die Evaluation gelangte ausserdem zu dem Ergebnis, dass durch die CARO-Lernumgebung eine intensivere Aktivierung aller Schülerinnen und Schülern stattfindet. Indem alle Schülerinnen und Schüler gleichermassen aufgefordert sind, Antworten oder Ergebnisse auf die Fragen der Lehrperson zu generieren, kann sich niemand entziehen. Darüber hinaus können Lehrpersonen einsehen, welche Antworten von welchen Schülerinnen und Schülern stammen und gewinnen darüber wichtige Informationen für die Lernstandsdiagnostik.

Gleichzeitig kann diese hohe Aktivierung aber auch zu einer Überforderung der Lehrperson führen. Liegen von mehr als zwanzig Schülerinnen und Schüler jeweils mehrzeilige Antworten vor, ist es nicht möglich, diese differenziert im Unterrichtsgespräch zu bearbeiten. Für das Re-Design wurde daraus geschlussfolgert, dass, sofern eine Bearbeitung im Plenum intendiert ist, die Antworten entweder standardisiert oder aber auf wenige Schlagworte beschränkt werden müssen und ggf. im mündlichen (analogen) Unterrichtsgespräch vertiefend besprochen werden müssen.

Wie sich in der Evaluation der CARO-Lernumgebung gezeigt hat, müssen bei der unterrichtlichen Interaktion die jeweiligen Potenziale und Grenzen der analogen und der digitalen Interaktion berücksichtigt werden. Das beschriebene Phänomen bezeichnet Jahnke (2015, 47 ff.) als «Co-expanding Communication Spaces». Durch Integration der onlinebasierten Lernumgebung in den Pflegeunterricht erweitert sich die (analoge) Präsenzinteraktion. Werden im Moment in der CARO-Lernumgebung 
noch sehr begrenzte Interaktionsformate genutzt, die grösstenteils wenn auch mit etwas höherem Aufwand - ebenso mit analogen Methoden bewerkstelligt werden könnten, so ist geplant, den Kommunikationsraum zukünftig noch stärker auszudehnen und zu dynamisieren, indem Lernaufgaben in der beruflichen Praxis abzuleisten sind, weitere Personen, die sich ausserhalb des Klassenzimmers befinden, oder Netzwerke und Communities zu Lernzwecken aktiv eingebunden werden. Die Erkenntnisse zur Verknüpfung von digitaler und analoger Interaktion betreffen vorerst stärker die Nutzung der CARO-Lernumgebung als die Entwicklung und wurden daher in das CARO-Schulungskonzept integriert.

\subsection{Construction: Lern- und Erkenntnisprozesse durch pflege- und mediendidaktische Feinabstimmung digital unterstützen}

Ein grundlegendes Prinzip der Interaktionistischen Pflegedidaktik besteht darin, die Unterrichtsgegenstände in Kombination mit den für sie spezifischen Erkenntnismethoden zu lehren und lernen. Bezogen auf die Konzeption des CARO-Unterrichts wurde im Rahmen des ersten Feldtests festgestellt, dass hierfür eine stärkere Verschränkung pflege- und mediendidaktischer Ansätze erforderlich ist. Für die verschiedenen pflegedidaktischen Erkenntnisdimensionen muss das CARO-System jeweils geeignete methodische Tools und Interaktionsformate zur Verfügung stellen, mit denen die Schülerinnen und Schüler die intendierten Kompetenzen konstruieren können. Umgekehrt können aufgrund des bislang begrenzten Umfangs an vorhandenen Interaktionsformaten pflegedidaktische Ziele nicht vollumfänglich umgesetzt werden. Die enge Verknüpfung der pflegeund mediendidaktischen Gestaltung wird am Beispiel einer Unterrichtssequenz erläutert, in der die Schülerinnen und Schüler widersprüchliche Anforderungen in der Versorgung von Menschen mit Demenz (im Krankenhaus) reflektieren sollen.

Die Interaktionistische Pflegedidaktik zielt darauf ab, dass die Schülerinnen und Schüler, in der Pflege Phänomene von Misshandlung, Entrechtung und Entwürdigung (Honneth 1992, 212) erkennen, reflektieren und solche Handlungsalternativen wählen, die zu einem Abbau, einer 
Abschwächung oder einem Ausserkraftsetzen dieser Phänomene führen. Um Routinen, Ideologien und Gewohnheiten zu hinterfragen, wird auf das dialektische Verfahren des Denkens in Widersprüchen zurückgegriffen (Danner 2006, 193 ff.; Darmann-Finck und Duveneck 2018). Durch das Aufstellen einer These und das anschliessende systematische Infragestellen in Form einer Gegenthese, ergibt sich das kritische Potenzial dieser Methode. Der Widerspruch bzw. das Gegenargument werden damit positiv als Gelegenheit zur Weiterentwicklung des aktuellen Erkenntnisstands bzw. zur «Wahrheitsfindung» genutzt (Danner 2006, 211). Das Wechselspiel von These und Gegenthese führt zu einer Synthese, was nicht bedeuten muss, dass der Gegensatz tatsächlich aufgehoben ist, sondern dass These und Antithese auf einer abstrakteren Ebene zusammengeführt werden. Widersprüche müssen nicht, aber können faktisch vorliegen. Wenn sie faktisch vorliegen, hat die dialektische Methode einen besonderen Nutzen, da Widersprüche den Pflegerinnen und Pfleger oftmals nicht bewusst sind und daher einseitig (mit der Folge von Misshandlung, Entrechtung und Entwürdigung) aufgelöst werden. Durch die Anwendung des dialektischen Verfahrens können sie bewusst gemacht, akzeptiert und es können gezielt Formen des adäquaten Umgangs gesucht werden.

Anhand der Unterrichtseinheit «Besondere Ungewissheit im pflegerischen Handeln am Beispiel der Pflege von Menschen mit Demenz» soll der kontradiktorische Widerspruch zwischen Begründungsverpflichtung und Nichtwissen bzw. Entscheidungszwang erarbeitet werden. Pflegefachpersonen sind aufgrund ihrer Berufsausbildung verpflichtet, ihr Handeln auf gesichertes und nachprüfbares Begründungswissen zu stützen. Zugleich müssen pflegerische Handlungen aber der Situation entsprechen, wie sie sich aus der einzigartigen Perspektive der jeweiligen zu pflegenden Menschen darstellt. Solche lebenspraktischen Entscheidungen sind aufgrund der Zukunftsoffenheit jeder Lebenspraxis stets ungewiss. Diese Ungewissheit, die in jeder Pflegesituation besteht, verstärkt sich bei Menschen mit Demenz, da professionell Pflegende nie ganz sicher sein können, ob sie die Bedürfnisse der zu pflegenden Menschen auch tatsächlich korrekt erfasst haben (vgl. Evers 2012). 
Bei der Konzeption der Unterrichtseinheit mit der CARO-Lehr-/Lernumgebung bestand die Herausforderung darin, Interaktionsformate zu generieren, anhand derer das dialektische Verfahren umgesetzt werden kann. Die nun beispielhaft dargestellte Unterrichtssequenz gliedert sich in drei Phasen:

I - Entscheidung provozieren mittels einer zweipoligen Abfrage Mit zweipoligen Abfragen werden die Schülerinnen und Schüler angeregt, sich zwischen einer der beiden Alternativen zu entscheiden. Eine solche Abfrage kann als Einstieg in die Bearbeitung von Widersprüchen genutzt werden, um das Vorverständnis der Lernenden transparent zu machen und im Anschluss daran anknüpfen zu können. Auf eine solche Abfrage hin werden sich einige Schülerinnen und Schüler ohne Nachfrage zuordnen, andere werden vermutlich mit Widerstand reagieren («Ich kann mich gar nicht entscheiden»). In der im Mittelpunkt stehenden Sequenz können die Schülerinnen und Schüler auf die über die App erhaltene Frage «Ist Ausprobieren unprofessionell?» mit «ja» oder «nein» antworten. Diese Frage richtet sich auf einen Pol des Widerspruchs, nämlich die Ungewissheit, die es erfordert, sich mit einer offenen Wahrnehmungseinstellung in einem hermeneutischen Prozess der Lebenspraxis und Perspektive der zu pflegenden Menschen anzunähern (hier zunächst laienhaft als «Ausprobieren» bezeichnet). Die Lehrperson fordert die Schülerinnen und Schüler auf, sich für die Alternative zu entscheiden, der sie eher zustimmen würden und wählt in der «CARO Lehrer*innen App» eine entsprechende Visualisierungsform für das Abstimmungsergebnis aus (z. B. Tortendiagramm). Auf der Basis des Abstimmungsergebnisses kann die weiterführende Frage, welche Argumente für die eine oder die andere Position sprechen, zunächst kurz mündlich diskutiert werden.

II - Wechselspiel von These und Gegenthese anhand von Arbeitsaufträgen mit vertiefenden Fragen und Texten

Zur Vertiefung der bereits in der Diskussion aufgeworfenen Argumentationslinien erhalten die Schülerinnen und Schüler im nächsten Schritt über die «CARO Schüler*innen App» drei 
Gruppenarbeitsaufträge. Die einzelnen Aufträge werden erst nach Vorliegen einer Antwort zu dem vorausgegangenen Auftrag freigeschaltet. Dabei werden die beiden Aussagen «Ausprobieren ist unprofessionell» mit dem Anspruch einer gut abgesicherten Begründung und «Ausprobieren ist professionell» mit dem Anspruch, sich an der Lebenspraxis der zu pflegenden Person zu orientieren, nacheinander mittels kleiner Texte und offener Fragen, auf die die Schülerinnen und Schüler mit Freitexten antworten sollen, mit pflegewissenschaftlichen Begründungen hinterlegt. Da die Ergebnissicherung bei Freitexten über das Präsentationsboard unpraktikabel ist, muss die Ergebnissicherung nach Abschluss der Gruppenarbeitsphase entweder mündlich im Unterrichtsgespräch erfolgen oder aber die Schülerinnen und Schüler werden gebeten, die Ergebnisse zu jeder Aufgabe auf eine bestimmte Zeichenzahl zu komprimieren. Die komprimierten Freitexte können dann auf dem Whiteboard gezeigt und im Plenum interpretiert werden. Die gemeinsame Deutung führt zu der Erkenntnis, dass in der Pflege beide Ansprüche gleichermassen zu berücksichtigen sind.

III - Zusammenfassung einer zentralen Erkenntnis anhand eines selbstproduzierten Expertinnen- bzw. Experteninterviews

Um das Lernergebnis zu sichern, erhalten die Schülerinnen und Schüler über die «CARO Schüler*innen App» die Aufgabe, in Kleingruppen ein kurzes Expertinnen- bzw. Experteninterview (max. 5 Min.) zum Thema «Ist Ausprobieren unprofessionell?» aufzunehmen. Das Format «Expertinnen- bzw. Experteninterview» fordert dazu auf, ein fachlich gut begründetes und ausgewogenes Statement zu produzieren. Die verschiedenen Interviews werden den Arbeitsgruppen im Anschluss zur Kommentierung zur Verfügung gestellt. Sie erhalten den Auftrag, Gemeinsamkeiten und Unterschiede festzustellen. Die ermittelten Ergebnisse werden abschliessend auf dem Whiteboard präsentiert und genutzt, um erneut herauszustellen, dass der Widerspruch letztlich nicht auflösbar ist und daraus Schlussfolgerungen für das pflegerische Handeln abzuleiten. Die Lehrerperson könne im Anschluss als mögliche Handlungsoption in der Versorgung von Menschen mit Demenz (im Krankenhaus) 
anhand eines PPT-gestützten Lehrpersonenvortrags die Serial Trial Intervention (STI) vorstellen (Hennig 2018), eine Intervention, bei der systematisch (auf der Basis pflegewissenschaftlichen Wissens) verschiedene Hintergründe einer Unruhe seitens der zu pflegenden Person mit Demenz versuchsweise überprüft ("ausprobiert») und ausgeschlossen werden.

In der beschriebenen Unterrichtseinheit wurden, um das Bildungsziel, den Widerspruch zwischen Begründungsverpflichtung und Nichtwissen bzw. Entscheidungszwang transparent zu machen und tiefergehend zu verstehen, eine zweipolige standardisierte Abfrage, offene Abfragen mit der Möglichkeit, Freitexte einzugeben, die eigene Produktion von Videos sowie Kommentarfunktionen genutzt. Für das Re-Design wurde hieraus die Konsequenz abgeleitet, zukünftig weitere Interaktionsformate, mit denen sich Widersprüche oder andere Bildungsziele bearbeiten lassen, bereitzustellen. An diesem Beispiel wird deutlich, dass die digitalen Interaktionsformate an sich noch kein Bildungspotenzial beinhalten, vielmehr erschliesst sich dieses erst in Kombination mit einem Lerngegenstand. Anhand der methodisch gestützten Bearbeitung des Lerngegenstands generieren die Schülerinnen und Schüler Erkenntnisse. Die pflege- und mediendidaktische Feinabstimmung ist dabei nicht nur für die Gestaltung einzelner Unterrichtseinheiten von Bedeutung. Es eröffnet sich auch die Aussicht und das Desiderat, eine auf die pflegedidaktischen Bildungsdimensionen abgestimmte Systematik digitaler Werkzeuge im Sinne einer Fachmediendidaktik weiter auszudifferenzieren und theoretisch zu untermauern.

\section{Ausblick}

Da in dem Vorhaben zunächst die Entwicklung der CARO Lehr-/Lernumgebung im Mittelpunkt stand, richtete sich die Evaluation primär auf die Ermittlung von Optimierungsmöglichkeiten des «digitalen» didaktischen Designs. Das zentrale Ziel von CARO ist eine möglichst hohe Aktivierung der Lernenden, um tiefgehende Lernprozesse anzuregen. Wie bereits in der Evaluation erprobt, unterstützt die CARO Plattform die digitale 
Umsetzung verschiedener methodischer Ansätze wie Exposition, Exploration, Problemorientierung sowie Kooperation (vgl. Kerres 2018). Dabei konnten verschiedene Formen der zeitlichen, räumlichen und sozialen Organisation realisiert werden.

Neben zahlreichen Optimierungsmöglichkeiten konnten weitere Lernpotenziale festgestellt werden. Neben dem Aufbau von Pflegekompetenz ermöglicht die CARO Lehr-/Lernumgebung die Förderung unterschiedlicher Komponenten von Medienkompetenz bzw. Medienbildung (BMBF - Bundesministerium für Bildung und Forschung 2010; KMK - Kultusministerkonferenz 2016). So stellt die Lehr-/Lernumgebung CARO selbst eine Form der digitalen Wirklichkeit dar, bei der digitale Formate genutzt werden, um die Interaktion im Klassenraum und Bildungsprozesse zu gestalten. Dadurch lassen sich Chancen und Risiken von digitalisierten Prozessen exemplarisch untersuchen. Die Schülerinnen und Schüler werden ausserdem angeregt, unterschiedliche multimediale Ausdrucksformen (kreativ) zu gestalten. Durch die Rezeption von Filmen, werden unterschiedliche Interpretationen von Pflegesituationen ermöglicht und das (auch) experimentelle und spielerische Entwerfen alternativer Sichtweisen durch die Produktion neuer Filme gefördert. So werden eigene Rezeptionsgewohnheiten bewusst und alternative Entwürfe erprobt, die auch für die eigene Persönlichkeitsentwicklung wirksam sein können. Sie nutzen die Lernumgebung des Weiteren, um mit- und voneinander zu lernen.

Damit das didaktische Design die hier vorgestellten Potenziale entfalten kann, müssen seitens der Schulen einige technische Voraussetzungen und Rahmenbedingungen gegeben sein. Ein stabiles, für die Lehrpersonen und Schülerinnen und Schüler barrierearm und zugleich sicher zugängliches WLAN mit guter Übertragungsleistung ist die zentrale Voraussetzung für den digitalen Datentransfer. Für die Lehrpersonen müssen geeignete Geräte für die Vorbereitung und Durchführung von der Einrichtung zur Verfügung gestellt werden. Bei den Schülerinnen und Schülern kann hingegen mit hoher Wahrscheinlichkeit davon ausgegangen werden, dass diese über ein mobiles Endgerät verfügen. Um die Zugänglichkeit zu den digitalen Lernformen gleichermassen für alle Schülerinnen und Schülern zu ermöglichen, müssen gegebenenfalls einzelne Geräte von der Schule vorgehalten werden. Sowohl bei den Lehrpersonen als auch bei den 
Schülerinnen und Schülern können die Einstellungen und Haltungen zur Nutzung von digitalen Medien für Lernprozesse sehr unterschiedlich sein. Einerseits bestehen erhebliche Unsicherheiten im Umgang mit digitalen Medien und eine Zurückhaltung beim Einsatz digitaler Medien im Unterricht. Andererseits gibt es Interesse, Neugierde sowie Offenheit und Bereitschaft, digitale Medien im Unterricht zu nutzen.

Nur im Zusammenspiel der «Rahmenbedingungen», der «didaktischen Interaktionen» sowie des «didaktischen Designs» kann das Potential digitaler Lernformate bestmöglich ausgeschöpft werden (Jahnke 2015, 134). Wurden die Unterrichtseinheiten im Projekt CARO anfangs vorwiegend ausgehend vom Pflegepräsenzunterricht und den üblichen analogen $\mathrm{Me}$ thoden konzipiert, weitete sich der Fokus im Verlauf des Projekts und es wurden zunehmend Perspektiven der Mediendidaktik (Kerres 2018; Wolf 2003) aufgenommen. Eine systematische, theoriebasierte Verschränkung des pflegedidaktischen Ansatzes der Interaktionistischen Pflegedidaktik und der digitalen Didaktik steht aber noch aus.

\section{Förderung}

Das Projekt CARO wird gefördert durch das BMBF und dem ESF mit dem Förderkennzeichen 01PD15012. 


\section{Literatur}

Akker, Jan van den, Koeno Gravemeijer, Susan McKenney, und Nienke Nieveen, Hrsg. 2006. Educational design research. London; New York: Routledge.

Arnold, Rolf. 1990. Berufspädagogik: Lehren und Lernen in der beruflichen Bildung; ein Studienbuch. 1. Aufl. Bd. 17. Berufspädagogik bei Sauerländer und Diesterweg. Aarau: Verl. für Berufsbildung Sauerländer.

Birman, Ken P., und Thomas A. Joseph. 1987. «Exploiting Virtual Synchrony in Distributed Systems». In Proceedings of the Eleventh ACM Symposium on Operating Systems Principles - SOSP '87, 123-38. Austin, Texas, United States: ACM Press. https://doi.org/10.1145/41457.37515.

BMBF - Bundesministerium für Bildung und Forschung, Hrsg. 2010. Kompetenzen in einer digital geprägten Kultur. Medienbildung für die Persönlichkeitsentwicklung, für die gesellschaftliche Teilhabe und für die Entwicklung von Ausbildungs- und Erwerbsfähigkeit. Berlin: Bundesministerium für Bildung und Forschung (BMBF) Referat Digitale Medien und Informationsinfrastruktur. http://www.dlr.de/pt/Portaldata/45/Resources/a_dokumente/bildungsforschung/Medienbildung_Broschuere_2010.pdf.

Cao, Bingyi, Margarita Esponda-Argüero, und Raul Rojas. 2016. «Development and Evaluation of a Classroom Interaction System». In Proceedings of the International Association for Development of the Information Society (IADIS), herausgegeben von Inmaculada Arnedillo Sánchez und Pedro Isaías, 59-66. Vilamoura, Algarve, Portugal: IADIS. http://www.iadisportal.org/digital-library/ mdownload/development-and-evaluation-of-a-classroom-interaction-system.

Danner, Helmut. 2006. Methoden geisteswissenschaftlicher Pädagogik: Einführung in Hermeneutik, Phänomenologie und Dialektik; mit ausführlichen Textbeispielen. 5., Überarb. und erw. Aufl. UTB Geisteswissenschaften 947. München: Reinhardt.

Darmann-Finck, Ingrid. 2010. Interaktion im Pflegeunterricht: Begründungslinien der interaktionistischen Pflegedidaktik. Bd. 1. IPP-Pflegeforschung. Frankfurt am Main: Peter Lang.

Darmann-Finck, Ingrid, und Nicole Duveneck. 2018. "Forschendes Lernen am Beispiel eines multimedialen Lernangebots zur Pflege von Menschen mit Demenz». Pädagogik der Gesundheitsberufe 3: 24-33. https://docplayer. org/108126063-Paedagogik-der-gesundheitsberufe.html.

Deutsches Netzwerk für Qualitätsentwicklung in der Pflege (DNQP), Hrsg. 2019. «Expertenstandard Beziehungsgestaltung in der Pflege von Menschen mit Demenz: Einschließlich Kommentierung und Literaturstudie». Hochschule Osnabrück. https://www.dnqp.de/fileadmin/HSOS/Homepages/DNQP/Dateien/Expertenstandards/Demenz/Demenz_AV_Auszug.pdf.

Evers, Thomas. 2012. Die besondere Ungewissheit im Handeln. Peter Lang D. https:// doi.org/10.3726/978-3-653-01892-9. 
Flick, Uwe, Ernst von Kardorff, und Ines Steinke, Hrsg. 2017. Qualitative Forschung: ein Handbuch. 12. Auflage, Originalausgabe. Rororo Rowohlts Enzyklopädie 55628. Reinbek bei Hamburg: rowohlts enzyklopädie im Rowohlt Taschenbuch Verlag.

Habermas, Jürgen. 2001. Erkenntnis und Interesse: mit einem neuen Nachwort. 13. Aufl. Suhrkamp-Taschenbuch Wissenschaft 1. Frankfurt am Main: Suhrkamp.

Hepp, Andreas. 2018. «Von der Mediatisierung zur tiefgreifenden Mediatisierung. Konstruktivistische Grundlagen und Weiterentwicklungen in der Mediatisierungsforschung». In Kommunikation - Medien - Konstruktion. Braucht die Mediatisierungsforschung den Kommunikativen Konstruktivismus?, herausgegeben von Jo Reichertz und Richard Bettmann, 27-45. Wissen, Kommunikation und Gesellschaft. Wiesbaden: Springer VS. https://doi.org/10.1007/978-3-65821204-9_2.

Hennig, Andre. 2018. «Bedürfnisorientierung ist der Schlüssel». Die Schwester/ Der Pfleger 57 (3): 22-27. https://www.bibliomed-pflege.de/sp/artikel/34557beduerfnisorientierung-ist-der-schluessel.

Honneth, Axel. 1992. Kampf um Anerkennung: zur moralischen Grammatik sozialer Konflikte. 1. Aufl. Frankfurt am Main: Suhrkamp.

Jahnke, Isa. 2015. Digital Didactical Designs: Teaching and Learning in CrossActionSpaces. 1. Aufl. New York; London: Routledge, Taylor \& Francis Group. https://doi.org/10.4324/9781315681702.

Kamin, Anna-Maria. 2013. Beruflich Pflegende als Akteure in digital unterstützten Lernwelten. Wiesbaden: Springer Fachmedien Wiesbaden. https://doi. org/10.1007/978-3-658-02310-2.

Kelle, Udo, und Christian Erzberger. 2007. "Qualitative und quantitative Methoden: kein Gegensatz». In Qualitative Forschung: Ein Handbuch, 4. Auflage, 384-401. Hamburg: Rowohlt.

Kerres, Michael. 2018. Mediendidaktik: Konzeption und Entwicklung digitaler Lernangebote. 5. Auflage. Berlin; Boston: De Gruyter Oldenbourg. https://doi. org/10.1515/9783110456837.

Kerres, Michael, und Claudia de Witt. 2003. «A Didactical Framework for the Design of Blended Learning Arrangements». Journal of Educational Media 28 (2-3): 101-13. https://doi.org/10.1080/1358165032000165653.

Klafki, Wolfgang. 1994. Neue Studien zur Bildungstheorie und Didaktik: zeitgemäße Allgemeinbildung und kritisch-konstruktive Didaktik. 4. Auflage. Weinheim Basel: Beltz Verlag.

KMK - Kultusministerkonferenz. 2016. «Bildung in der digitalen Welt. Strategie der Kultusministerkonferenz». Herausgegeben von Sekretariat der Kultusministerkonferenz. Kultusministerkonferenz. https://www.kmk.org/fileadmin/ Dateien/pdf/PresseUndAktuelles/2017/Digitalstrategie_KMK_Weiterbildung. pdf. 
Knoll, Jörg. 2007. Kurs- und Seminarmethoden: ein Trainingsbuch zur Gestaltung von Kursen und Seminaren, Arbeits- und Gesprächskreisen. Neu ausgestattete Sonderausg. der 11. Aufl. Bd. 2. Basisbibliothek für Seminare und Trainings. Weinheim: Beltz.

Koppel, Ilka. 2016. Entwicklung einer Online-Diagnostik für die Alphabetisierung. Wiesbaden: Springer Fachmedien Wiesbaden. https://doi.org/10.1007/978-3658-15769-2.

Kuckartz, Udo. 2016. Qualitative Inhaltsanalyse: Methoden, Praxis, Computerunterstützung. 3., Überarbeitete Auflage. Grundlagentexte Methoden. Weinheim Basel: Beltz Juventa.

Mayring, Philipp, Hrsg. 2015. Qualitative Inhaltsanalyse: Grundlagen und Techniken. 12., Überarb. Aufl. Weinheim: Beltz.

Mehan, Hugh. 1979. Learning Lessons: Social Organization in the Classroom. Cambridge, Massachusetts and London, England: Harvard University Press. https://doi.org/10.4159/harvard.9780674420106.

Meteor Development Group. 2019. "Publish and subscribe. Documentation of Meteor's publication and subscription API». 2019. https://docs.meteor.com/ api/pubsub.html.

Meyer, Meinert A. 2008. Perspektiven der Didaktik. Bd. 9. Zeitschrift für Erziehungswissenschaft. Sonderheft. Wiesbaden: VS Verlag für Sozialwissenschaften. https://doi.org/10.1007/978-3-531-91775-7.

Prince, Michael. 2004. «Does Active Learning Work? A Review of the Research». Journal of Engineering Education 93(3): 223-231. https://doi. org/10.1002/j.2168-9830.2004.tbo0809.x.

Voigt, Jörg. 1990. «Die interaktive Konstitution fachlicher Themen im Unterricht: Zum Einfluß von Alltagsvorstellungen». In Zur Didaktik der Physik und Chemie, 74-88. Alsbach/Bergstr.: Leuchtturm.

Weidenmann, Bernd. 2015. Handbuch Active Training: die besten Methoden für lebendige Seminare. 3., Aktualisierte und Erweiterte Auflage. Beltz Weiterbildung - Qualifikation. Weinheim Basel: Beltz.

Wolf, Karsten D. 2003. Gestaltung und Einsatz einer internetbasierten Lernumgebung zur Unterstützung Selbstorganisierten Lernens. Bd. 1. Medienpädagogik und Mediendidaktik. Hamburg: Kovač. 